\title{
Use of contraceptives and uptake of long-acting reversible contraception among postpartum women in rural China
}

\author{
Chuyun Kang, ${ }^{1}$ Peng Li, ${ }^{1}$ Xiaoli Liu, ${ }^{1}$ Yuan Ding, ${ }^{1}$ Xiaoye Wang, ${ }^{2}$ \\ Hong Zhou ${ }^{1}$
}

- Additional material is published online only. To view please visit the journal online (http://dx.doi.org/10.1136/ bmjsrh-2017-101849).

1 Department of Maternal and Child Health, School of Public Health, Peking University, Beijing, China

${ }^{2}$ Gynaecology and Obstetrics Department, Peking University, Third Hospital, Beijing, China

\section{Correspondence to}

Professor Hong Zhou, Department of Maternal and Child Health, School of Public Health, Peking University, Beijing, 100191, China; hongzhou@ bjmu.edu.cn and Dr Xiaoye Wang, Gynaecology and Obstetrics Department, Peking University Third Hospital, Beijing, 100191, China; wxydg2007@ 126.com

CK and PL contributed equally.

Received 12 June 2017 Revised 31 May 2018 Accepted 19 June 2018 Published Online First 6 August 2018
Check for updates

(C) Author(s) (or their employer(s)) 2018. No commercial re-use. See rights and permissions. Published by BMJ.

To cite: Kang C, Li P, Liu X, et al. BMJ Sex Reprod Health 2018;44:254-259.

\begin{abstract}
Background Pregnancies have been increasing since the universal two-child policy in China was announced and thus the prevention of short inter-pregnancy intervals has become more important. However, little is known about contraceptive use among postpartum women in rural areas of China. This study investigated the current situation and factors associated with contraceptive use, especially long-acting reversible contraception (LARC) practice among postpartum women.
\end{abstract}

Study design A cross-sectional study was conducted with a probability proportionate to size (PPS) sampling method in Shimen County of Hunan province with 423 mothers whose youngest child was aged under 2 years. A questionnaire was used to collect information on participants' demographics, reproductive history and contraceptive use.

Results The rate of using all contraceptive methods was $66.4 \%$ in the study group and the rate of using LARC was $9.9 \%$. Statistically significant predictors of contraceptive use were: no breastfeeding, no intention of having another child, and education beyond high school. Statistically significant predictors of LARC utilisation were: vaginal delivery, at least one child before the index delivery, and no breastfeeding.

Conclusions The utilisation rate of postpartum contraception, especially LARC, was relatively low in rural China. The benefits of LARC have not been realised and breastfeeding is still incorrectly believed to be a definitely reliable method of contraception. Thus, health education and contraceptive knowledge and regulations need to be updated and enhanced based on research evidence, for increased adoption of postpartum contraception in rural China.

\section{Key messages}

Nearly two-thirds of postpartum women were using contraceptives and no more than one-fifth of them chose long-acting reversible contraception.

- Breastfeeding is still wrongly believed to be a definitely reliable method of contraception.

- National programme strategies, such as counselling or education about postpartum family planning, are required to be improved to increase the adoption of postpartum contraception in rural China.

\section{INTRODUCTION}

According to WHO recommendations, after a live birth the interval before attempting the next pregnancy should be at least 24 months in order to reduce the risk of adverse maternal, perinatal and infant outcomes. ${ }^{1}$ Since 2015, China's one-child policy has been replaced by a universal two-child policy. Consequently, the number of pregnancies has been increasing under the new fertility policy, emphasising awareness and importance of preventing rapid subsequent pregnancies.

Among contraceptive methods, longacting reversible contraceptive (LARC) methods, including intrauterine devices (IUDs) and contraceptive implants, are user-independent, are safe and have very low failure rates (less than 1\%), which is comparable to those with sterilisation. ${ }^{2}$ LARC, therefore, has been identified as a key priority in the national strategic plans for family planning in many countries. ${ }^{3}$

In China, awareness of the importance of postpartum contraception and the 
provision of family planning services after delivery and during the first several years postpartum have remained insufficient. The incidence of unintended pregnancy was 3.8 per 100 women-years, which is associated with an increased risk of induced abortion and rapid repeat pregnancy. ${ }^{4}$ Data from the National Population and Family Planning Commission of the People's Republic of China (NPFPC) suggested that Chinese women seldom choose contraceptive implants, diaphragm contraceptives, cervical caps, the rhythm method and spermicide for contraception; as a consequence the prevalence rate of all of these declined from $0.55 \%$ in 1990 to $0.03 \%$ in $2010 .^{5}$

One important step in promoting the utilisation of family planning services in China is to explore factors that may encourage women to use contraceptives. To date, very few studies in China have explored contraceptive use specifically targeting the postpartum period, and even fewer in rural settings. Therefore, the present study was designed to investigate the pattern of contraceptive use after delivery and the determinants of uptake of contraceptives and LARC usage among rural postpartum Chinese women.

\section{METHODS}

\section{Subjects and sampling}

This study used a part sample of a cross-sectional study which surveyed children under 3 years old conducted in Shimen county, Hunan province, in September 2015. This county had a population of 669741 with the per capita net income of rural residents of Yuan 8778 in 2015, which was lower than the national average (Yuan 10 772). ${ }^{67}$

The sample size of mothers was determined based on the assumptions of a proportion of contraceptive use of $50 \%$ and a tolerance error of $5 \%$. The calculated minimum sample size was 384 .

In the survey, participants were selected using a three-stage sampling procedure. The population sizes of each administrative and natural village used for sampling were provided by the county health bureau. In the first stage, 15 administrative villages were selected with probability proportional to size (PPS). In the second stage, two natural villages were selected with PPS in each of the sampled administrative villages. In the third stage, 16 mothers were randomly selected in each of the selected natural villages, using the name list of households with children under 3 years old collected by village doctors. Finally, 503 mothers were recruited for the study. According to WHO recommendations of at least 24 months before attempting the next pregnancy, ${ }^{1} 423$ mothers of children under 2 years old among all mothers selected were used for analysis in this study.

The study was approved by the Ethics Committee of Peking University Health Science Centre. Informed consent was signed by each participant undertaking the interview.

\section{Patient and public involvement}

This study was a cross-sectional study with the subjects being healthy mothers of children under 2 years old. No patients were involved in this study.

\section{Study variables}

Face-to-face interviews were performed and a questionnaire was created for collecting information. Questionnaire items were adopted from 'Multiple Indicator Cluster Survey (MICS)' tools published by Unicef. ${ }^{8}$ Postpartum family planning was defined in the present study as contraception used up to 24 months after delivery.

The primary outcome was participants' contraception usage at the time of the survey, which was measured by the following question: 'Are you using any contraceptive methods now?'. This question was used in a survey of China's birth control among women of childbearing age, a study of the current family planning situation conducted by the National Health and Family Planning Commission (NHFPC). ${ }^{9}$ Participants who indicated that they were using contraceptives were asked which methods they adopted from the following options: intrauterine devices (IUDs), contraceptive implants, oral contraceptive pills, condom (male or female), sterilisation (male or female), withdrawal and rhythm method. The secondary outcome was the usage of LARC, including IUDs and implants. ${ }^{2}$

Potential factors associated with contraception use in the present study included demographic characteristics, delivery information, infant feeding practices and fertility intention. Demographic characteristics included: age $(<25,25-29, \geq 30$ years), education status (middle school and lower, high school, above high school), ethnicity (Han and others), and per capita annual household income $(0-3750,3751-$ 9999, $\geq 10000 \mathrm{RMB})$. Delivery information included: antenatal visits, gender of child (boy or girl), number of living children before index delivery $(0, \geq 1)$, and delivery mode (caesarean section or vaginal delivery). Antenatal visits were grouped into two categories: less than eight visits and eight or more, which was defined using WHO recommendations on antenatal care (ANC) ${ }^{10}$ The study used Unicef's MICS standard questionnaire to investigate breastfeeding status in the past 24 hours, including whether infants were given water, breast milk or any other foods. Infant feeding practice was classified into two categories: any breastfeeding and no breastfeeding. Finally, fertility intentions were assessed by asking whether or not they wanted another child.

\section{Statistical analysis}

Data were cleaned and analysed using Stata version 15.0 (College Station, TX: StataCorp LLC). To reflect the three-stage sampling design, Stata's survey procedures were used for all statistical tests. ${ }^{11}$ Chi-square test was used for bivariate analysis and the results were 
presented using crude OR and 95\% CIs. Multivariable logistic regression model was employed to identify potential factors associated with contraception use and LARC use. Adjusted ORs (aORs) with 95\% CIs were reported. Adjusted variables for contraceptive use and LARC use were age, education, ethnicity, per capita annual household income, antenatal visits, gender of last child, delivery mode, number of living children before index delivery, infant feeding and fertility intention. A P value less than 0.05 was set as the cut-off for statistical significance. Missing data were excluded from both Chi-square tests and logistic regression when the results showed no statistically significant differences for deleting that data.

\section{RESULTS}

\section{Characteristics of participants}

Among all the participants interviewed, 423 mothers had delivered within 24 months and were included in the analysis. Close to half only had middle school education or less (45.7\%). More than half (68.7\%) reported a total annual household income of less than Yuan 10000. More than half attended antenatal clinic visits at least eight times (57.9\%), 54.6\% of them had more than one child and $43.4 \%$ had delivered the youngest baby by caesarean section. One-third of the participants wanted to have another child. The percentage of mothers who were breastfeeding the last baby was $52.8 \%$ (table 1 ).

\section{Constitution of contraceptive method}

The overall prevalence of postpartum contraceptive use by women was $66.4 \%$. The most commonly used contraceptive method was condom, with $46.1 \%$ of women using it, accounting for $69.4 \%$ of contraception use (figure 1). Among all the stated contraceptive methods, the proportion of IUD use was only $14.9 \%$. There were no users of contraceptive implants. Thus the prevalence of using LARC was just 9.9\%.

\section{Factors relating to contraceptive usage}

The proportion of women who were not breastfeeding their baby who used contraception was $78.4 \%$ and significantly higher than the rate of $56.1 \%$ of women who were breastfeeding their baby $(\mathrm{aOR}=3.22$, $95 \% \mathrm{CI}=1.77$ to 5.85 ). Some $72.7 \%$ of women who did not desire another pregnancy were using contraception, compared with $52.6 \%$ of women who did $(\mathrm{aOR}=3.11$, $95 \% \mathrm{CI}=1.14$ to 8.51 ). Specifically, with all other variables being controlled, women with educational level above high school had significantly higher proportion of use of contraceptive methods than those with lower educational levels $(\mathrm{aOR}=2.57,95 \% \mathrm{CI}=1.08$ to 6.10$)$ (see online supplementary table 1 ).

\section{Factors relating to LARC method usage}

The prevalence of LARC use of women whose index delivery was vaginal was $12.9 \%$, significantly higher than the rate of $4.9 \%$ of women whose index birth was by

\begin{tabular}{|c|c|}
\hline Variables & Participants (n (\%)) \\
\hline \multicolumn{2}{|l|}{ Demographics } \\
\hline \multicolumn{2}{|l|}{ Age (years) } \\
\hline$<25$ & $91(21.5)$ \\
\hline $25-29$ & $174(41.1)$ \\
\hline$\geq 30$ & $158(37.4)$ \\
\hline \multicolumn{2}{|l|}{ Educational status } \\
\hline Middle school and less & $193(45.7)$ \\
\hline High school & $144(34.1)$ \\
\hline Above high school & $85(20.1)$ \\
\hline \multicolumn{2}{|l|}{ Ethnicity } \\
\hline Han & $210(49.6)$ \\
\hline Other & $213(50.4)$ \\
\hline \multicolumn{2}{|c|}{ Per capita annual household income (Yuan/person? } \\
\hline$\geq 10000$ & $131(31.3)$ \\
\hline $3751-9999$ & $190(45.5)$ \\
\hline $0-3750$ & $97(23.2)$ \\
\hline \multicolumn{2}{|l|}{ Delivery information } \\
\hline \multicolumn{2}{|l|}{ Antenatal clinic visits } \\
\hline$<8$ & $178(42.1)$ \\
\hline$\geq 8$ & $245(57.9)$ \\
\hline \multicolumn{2}{|l|}{ Gender of last child } \\
\hline Boy & $210(49.8)$ \\
\hline Girl & $212(50.2)$ \\
\hline \multicolumn{2}{|l|}{ Delivery mode } \\
\hline Caesarean & $182(43.4)$ \\
\hline Vaginal & $237(56.6)$ \\
\hline \multicolumn{2}{|c|}{ Number of living children before index delivery } \\
\hline 0 & $192(45.4)$ \\
\hline$\geq 1$ & $231(54.6)$ \\
\hline \multicolumn{2}{|l|}{ Current status } \\
\hline \multicolumn{2}{|l|}{ Infant feeding } \\
\hline Any breastfeeding & $219(52.8)$ \\
\hline No breastfeeding & $196(47.2)$ \\
\hline \multicolumn{2}{|l|}{ Wants another child } \\
\hline Yes & $121(30.9)$ \\
\hline No & $271(69.1)$ \\
\hline
\end{tabular}

caesarean section $(\mathrm{aOR}=3.50,95 \% \mathrm{CI}=1.40$ to 8.73$)$. There was also a significant difference of LARC proportion between women with at least one child before the index delivery and those with no previous children $(\mathrm{aOR}=5.40,95 \% \mathrm{CI}=1.95$ to 14.99$)$. The LARC use proportion of women who were not breastfeeding was $13.6 \%$, significantly higher than the rate of $5.6 \%$ of women who were breastfeeding $(\mathrm{aOR}=3.93$, $95 \% \mathrm{CI}=1.51$ to 10.25 ) (see online supplementary table 2). 


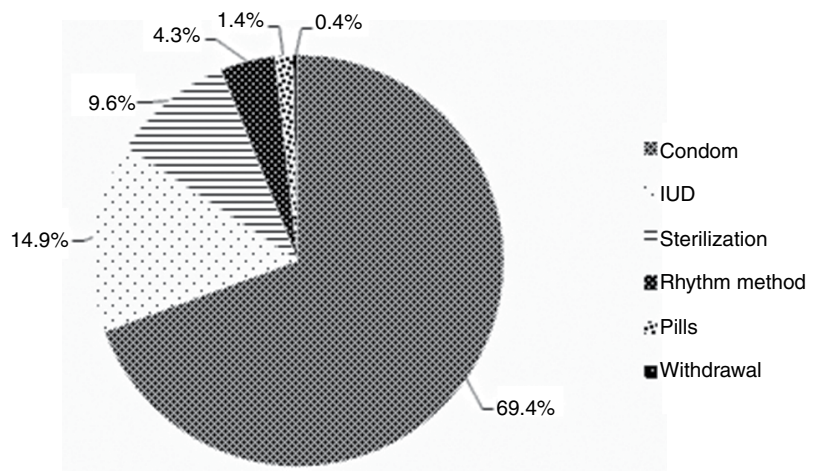

Figure 1 Constitution of postpartum contraceptive method. Percentage of postpartum women using intrauterine devices (IUD), condom, sterilisation, rhythm method, contraceptive pills and withdrawal at the time of investigation.

\section{DISCUSSION}

The present study contributes to our understanding of the prevalence of postpartum family planning among mothers with children under 2 years old, and identifying the determinants of uptake of contraceptives in rural China. In the present study, $66.4 \%$ of the women used contraception during the 2 years following delivery. The fact that more than $30 \%$ of postpartum women stated that they were using no method of contraception indicated their risk of unwanted pregnancy, which contributes to the incidence of dangerously short inter-pregnancy intervals. From the study, the factors associated with contraceptive utilisation were higher education level, no breastfeeding status, and no intention of having another child. In addition, vaginal delivery, no breastfeeding and having at least one child before the index delivery were associated with use of IUDs.

A national study in the USA showed that $76 \%$ of women who were 18 months post-delivery were using some form of contraceptive measures. ${ }^{12}$ In the present study, the prevalence rate of contraceptive usage of $66.4 \%$ among postpartum women in rural China was lower than not only the USA but also Chinese married women overall $\left(89.2 \%, 20-49\right.$ years). ${ }^{5}$ This result suggested that compared with all married women, postpartum women in rural China in similar situations need more attention regarding access to family planning information and services.

Among all contraceptive methods, condoms had the highest use rate of $46.1 \%$, which was much higher than the rate reported for all women of reproductive ages in China (9.3\%) in 2010. Although condom is classified as a less effective method in the 2015 publication Best practice in postpartum family planning, the WHO fact sheet on 'Family planning/contraception' states that if used correctly, male condoms have a 98\% effectiveness for preventing pregnancy as compared with $85 \%$ if only 'commonly' used. ${ }^{1314}$ For this reason, and as the most popular contraceptive method among postpartum couples, clear instructions on the correct and consistent use of condoms should be given to all couples.

In the present study, the rate of using IUDs was $9.9 \%$, which was much lower than the rate of $42.0 \%$ reported in the earlier study conducted in Shanghai by Huang. ${ }^{15}$ The overall use rate of LARC methods in our study was therefore $9.9 \%$ since no contraceptive implants were reported. Consistent with Hu's finding, despite the increasing need for LARC methods in China, there was a decreasing use of IUDs. ${ }^{16}$ In the USA, data from National Survey of Family Growth showed that LARC use increased $18.0 \%$ per year among postpartum women. ${ }^{17}$ The reason for the decreasing use of IUDs in China could be the relaxed reproductive policy which probably encourages individuals to have higher preference for short-term methods. ${ }^{5}$

Among the postpartum women we investigated, no contraceptive implants were used, which is similar to the rate of less than $2.6 \%$ reported by Huang. ${ }^{15}$ According to Chinese clinical practice guidelines, implants may cause changes in bleeding patterns, which could be the main reason for their very low use. ${ }^{18}$

The present study reinforces the importance of breastfeeding, which was a significant factor for both contraceptive use and IUD use. The major reason for nursing mothers not using any contraception is, on one hand, that breastfeeding delays the resumption of normal cycles, which offers relatively effective natural protection from conception. ${ }^{19}$ Conversely, mothers may become polarised by their beliefs that hormonal contraception has a negative impact on breastfeeding. ${ }^{20}$ In addition, as found by Rutaremwa et al, ${ }^{21}$ desire for another child was significantly associated with not using contraception during the extended postpartum period, which is possibly due to lack of knowledge about safe birth spacing.

The current study demonstrated a strong association between women's delivery mode and the utilisation of IUDs. Chinese Clinical Technology Operation Criteria for Family Planning published by the Chinese Medical Association in 2006 provides a recommendation that women should have an IUD inserted at least 6 months after caesarean birth, which may to some degree explain why women who had caesarean deliveries are less likely to use LARC. ${ }^{22}$ Research evidence suggests that postpartum and intrapartum insertion of IUDs is feasible and does not interfere with normal uterine involution. ${ }^{23}$ Hence, to insert IUDs immediately after the placenta has been delivered should be strongly recommended as best practice in China, as the overall caesarean prevalence is $34.9 \%{ }^{24}$ and $43.4 \%$ in the present study.

There are several limitations to this study. First, due to the constraints of time and funds, we could not carry out nationally representative sampling in this survey. 
One county, Shimen, cannot represent the entire rural areas of China.

Second, factors in postpartum contraceptive service provision were not considered in this study. For example, a survey of 209 obstetricians in Tianjin city showed that the correct rate of awareness of postpartum contraception among obstetricians was only $34.8 \% .^{25}$ The postpartum contraception services provided by these obstetricians were limited and could have an influence on use of contraception as well as on choosing contraceptive methods.

Third, some key factors that can have an impact on the use of postpartum contraceptives, such as resumption of menstruation and sexual behaviour post-delivery, were not considered in this study. Moreover, the lactational amenorrhea method (LAM) was not included as a postpartum contraceptive method option. One of the criteria for LAM, exclusive breastfeeding according to the Unicef definition, ${ }^{26}$ was so rare in rural China that in our study, among 125 mothers delivered within the previous 6 months, no women were exclusively breastfeeding.

In conclusion, approximately two-thirds of postpartum women were using contraception and more than two-thirds of them chose condoms, which focused on the importance of enhancing contraceptive use and improving the practice of LARC. From the findings, education level, no breastfeeding status, and no intention of having another child were related to contraceptive use among postpartum women. Particularly, having no children before the index delivery, breastfeeding and caesarean delivery were identified as restricting factors for IUD usage. Any programme aimed at promoting postpartum contraceptive use should target women of low educational level, breastfeeding mothers, and those intending to have another child. Empowering women through formal education, maintaining provision of health information, improving access to LARC methods, and providing guidance on the correct use of condoms, especially among groups at high risk of sexually transmitted diseases, during antenatal, delivery and postpartum periods are highly recommended.

\footnotetext{
Acknowledgements The authors would like to acknowledge Chinese Maternal and Child Health Association (CMCHA), Health and Family Planning Commission of Hunan Province for their participation in this research. The authors are very grateful to Prof. Ruyan Pang, Dr Luxia Gong of CMCHA, Dr Jiong Peng, Yanan Ren of the UNFPA and Shangchun Wu of the State Population and Family Planning Commission for technical assistance and guidance.
}

Funding This study was supported by the UNFPA/China CP7 project financed by United Nations Population Fund (UNFPA) China Country Office.

Competing interests None declared.
Patient consent Not required.

Ethics approval Ethics Committee of Peking University Health Science Center.

Provenance and peer review Not commissioned; externally peer reviewed.

\section{REFERENCES}

1 Marston C. Report of a technical consultation on birth spacing, Geneva, 13-15 June 2005. Geneva, Switzerland: World Health Organization, 2007.

2 Trussell J. Contraceptive failure in the United States. Contraception 2011;83:397-404.

3 Bakamjian L. Programming strategies for postpartum family planning. Geneva, Switzerland: World Health Organization, 2013.

4 Huang YM, Kang JZ, Xy H, et al. Study on unintended pregnancy and risk factors among postnatal women from inner city district in Shanghai. Reprod Contracept 2008;28:286-91.

5 Wang C. Trends in contraceptive use and determinants of choice in China: 1980-2010. Contraception 2012;85:570-9.

6 National statistical yearbook on economic and social development. 2015 http://www.stats.gov.cn/tjsj/zxfb/201602/ t20160229_1323991.html (accessed 26 Jan 2018).

7 Shimen statistical yearbook on economic and social development. 2015 http://www.shimen.gov.cn/Listzw/316.html (accessed 26 Jan 2018).

8 MICS6 Questionnaires. Secondary MICS6 questionnaires. http://mics.unicef.org/tools? round=mics6 (accessed 26 Jan 2018).

9 Li HGZ X, Wang Q. National population and family planning sampling investigation. Population Research 1990;4:24-6.

10 WHO. WHO recommendations on antenatal care for a positive pregnancy experience. Geneva Switzerland: WHO, 2016.

11 Stata Press. Stata survey data reference manual: release 13. College Station, TX: Stata Press, 2013.

12 White K, Teal SB, Potter JE. Contraception after delivery and short interpregnancy intervals among women in the United States. Obstet Gynecol 2015;125:1471-7.

13 Royal College of Obstetricians \& Gynaecologists. Best practice in postpartum family planning. London, UK: Royal College of Obstetricians \& Gynaecologists, 2015:2-7.

14 Hatcher RA, Trussell J, Stewart F, et al. eds. Contraceptive technology (18th revised edn). New York, NY: Ardent Media, 2004.

15 Huang YM, Merkatz R, Kang JZ, et al. Postpartum unintended pregnancy and contraception practice among rural-to-urban migrant women in Shanghai. Contraception 2012;86:731-8.

$16 \mathrm{Hu}$ X, Cheng L, Hua X, et al. Advanced provision of emergency contraception to postnatal women in China makes no difference in abortion rates: a randomized controlled trial. Contraception 2005;72:111-6.

17 Kavanaugh ML, Jerman J, Finer LB. Changes in use of longacting reversible contraceptive methods among U.S. women, 2009-2012. Obstet Gynecol 2015;126:917-27.

18 Chinese Medical Association. Clinical practice guidelines: Family Planning Volume[J]. China: Chinese Medical Association, 2005. 
19 Jackson E, Glasier A. Return of ovulation and menses in postpartum nonlactating women: a systematic review. Obstet Gynecol 2011;117:657-62.

20 King J. Contraception and lactation. J Midwifery Womens Health 2007;52:614-20.

21 Rutaremwa G, Kabagenyi A, Wandera SO, et al. Predictors of modern contraceptive use during the postpartum period among women in Uganda: a population-based cross sectional study. BMC Public Health 2015;15:262.

22 Chinese Medical Association. Chinese clinical technology operation criteria for family planning: People's Military Medical Press, 2006.
23 Zerzavy FM. Use of intrauterine contraceptive devices in the postpartum period. Am J Public Health Nations Health 1967;57:28-33.

24 Li HT, Luo S, Trasande L, et al. Geographic variations and temporal trends in cesarean delivery rates in China, 20082014. JAMA 2017;317:69-76.

$25 \mathrm{Xu} \mathrm{L}, \mathrm{Wu}$ S, Zhang A, et al. Cognition and attitude of postpartum contraception among obstetricians in Tianjin area[J]. Zhonghua fu chan ke za zhi 2014;49:842-6.

26 Labbok M, Cooney K, Coly S. Guidelines: breastfeeding family planning and the lactational amenorrhea method-LAM[J]: Washington DC, 1994. 